\title{
Prognostic factors of intraperitoneal chemotherapy for peritoneal carcinomatosis of gastric cancer: A retrospective study from a single center
}

\author{
HAI-TAO MEN $^{1,2^{*}}$, HONG-FENG GOU ${ }^{1 *}$, JI-YAN LIU ${ }^{1}$, QIU LI ${ }^{1}$, DE-YUN LUO ${ }^{1}$, FENG BI ${ }^{1}$ and MENG QIU ${ }^{1}$ \\ ${ }^{1}$ Division of Abdominal Cancer, Cancer Center, West China Hospital of Sichuan University, Chengdu, Sichuan 610041; \\ ${ }^{2}$ Department of Oncology, The First Affiliated Hospital of Chongqing Medical University, \\ Yuanjiagang, Yuzhong 400016, P.R. China
}

Received February 10, 2015; Accepted November 20, 2015

DOI: $10.3892 / \mathrm{ol} .2016 .4403$

\begin{abstract}
Peritoneal carcinomatosis (PC) of gastric origin is currently recognized as a terminal disease with a poor prognosis. Advancements in novel therapeutic approaches, including intraperitoneal chemotherapy (IPC), have recently been made and it is believed that this may have contributed to the improved survival observed in patients with PC. The present study aimed to investigate overall survival (OS) and the associated prognostic factors in patients with PC of gastric origin who underwent IPC. A total of 57 patients were studied, with a median age of 51 years. The median follow-up time was 12.4 months. PC was diagnosed in all patients with gastric cancer. The median survival time of all patients was 10.1 months, whilst the OS rate at 1,2 and 3 years was observed to be 46,19 and $12 \%$, respectively. Symptomatic ascites and a signet ring cell (SRC) histopathological type were demonstrated to signify a poor prognosis. Complete resection of all gross disease (CCR-0) and an increased number of cycles of systemic chemotherapy were independent factors that were observed to correlate with increased OS. The most common morbidities of grade 3/4 adverse effects were bone marrow suppression, nausea or vomiting, and diarrhea. In conclusion, IPC is an important treatment option for patients with PC that has originated from gastric cancer. Symptomatic ascites and SRC adenocarcinoma serve as negative clinicopathological prognostic factors, whilst CCR-0 and increased systemic chemotherapy cycles
\end{abstract}

Correspondence to: Dr Meng Qiu, Division of Abdominal Cancer, Cancer Center, West China Hospital of Sichuan University, 37 Guoxue Xiang Street, Chengdu, Sichuan 610041, P.R. China

E-mail: qiumeng33@hotmail.com

${ }^{*}$ Contributed equally

Key words: intraperitoneal chemotherapy, gastric cancer, peritoneal carcinomatosis, cytoreductive surgery
( $\geq 4$ cycles) may prove to be an important therapeutic option for PC patients.

\section{Introduction}

Gastric cancer is a prevalent disease, with the incidence rate being the fourth highest, and the mortality rate being the second highest, amongst all types of human cancer (1). The prognosis of advanced gastric cancer remains poor, with median survival time currently calculated at 7.9 months (2). The presence of peritoneal carcinomatosis (PC) is a poor prognostic factor in patients already suffering from advanced gastric cancer, functioning as a primary cause of mortality in nearly half of all patients (3). Until recently, no standardized effective treatment for PC originating from gastric origin had previously existed. Surgery alone has been demonstrated to provide no substantial benefit for prognosis, with a median survival time of 1 month (4). Therefore, surgery alone is not typically recommended as a treatment for patients with PC. Furthermore, systemic chemotherapy has also been proven to be ineffective against PC, with a median survival time of 6 months when used alone (5). Due to the effect of the peritoneal-blood barrier, when cytotoxic or biological agents are administered systemically, the concentration of such agents in the peritoneal cavity is less than one-third to one-fourth of the concentration observed in the blood and thus, numerous patients presenting with PC do not respond to systemic chemotherapy (6).

Intraperitoneal administration can deliver a higher concentration of cytotoxic or biological agents to the peritoneal cavity, [up to several fold higher compared with intravenous administration (7)], and can deliver them directly to the tumor site, therefore decreasing harmful systemic exposure. IPC is accepted as a standard treatment for gynecological tumors, resulting in marked improvements in patient survival (8). The efficacy of IPC in PC of gastric origin has not yet been fully understood, due to the rapid development of the disease and the short patient survival times. However, IPC has been considered as a potential therapy for PC $(1,9)$, with studies demonstrating a median survival time of 6.1-11.5 months after IPC treatment in patients with PC originating from gastric cancer $(3,10-12)$. Despite this, the 
median overall survival (OS) time of patients receiving IPC treatment varies, indicating that there may be confounding factors also affecting survival. Certain studies have reported favorable outcomes for patients who underwent a complete resection, including an improved physical status and the absence of ascites $(11,13,14)$. The present study reviewed the use of IPC in treating PC originating from gastric cancer, and aimed to identify any factors correlating with the effect of IPC.

\section{Materials and methods}

Study population. Patients with PC originating from gastric cancer received IPC treatment between June 2007 and October 2012, at the Cancer Center, West China Hospital of Sichuan University (Chengdu, China). All patients were confirmed to have synchronous peritoneal metastasis of gastric cancer upon histopathological analysis of surgery or biopsy specimens, or by analysis of the ascites cytology at first diagnosis. Criteria for eligible patients were as follows: Patients $\geq 18$ years of age, who presented with an adenocarcinoma of gastric origin [including signet ring cell (SRC) type] and who demonstrated no visceral metastasis (except ovarian metastasis). Patients had received at least one cycle of intraperitoneal chemotherapy and demonstrated an Eastern Cooperative Oncology Group (ECOG) score of 0-2 (15). Patients were excluded if they had a previous history of malignant disease or if they had previously received palliative chemotherapy. Patients were also excluded if they demonstrated the occurrence of peritonitis, intestinal obstruction, intestinal perforation or uncontrolled infection prior to treatment.

Data collection. Data regarding age, gender, ascites, ECOG performance status (PS) score, sites of primary disease, tumor differentiation, tumor markers [carcinoembryonic antigen (CEA), carbohydrate antigen 19-9 (CA19-9) and carbohydrate antigen 72-4 (CA72-4)] and stage of disease were collected. Symptomatic ascites was defined as a volume of $\geq 500 \mathrm{ml}$ identified during surgery or as estimated by computed tomography scan. Data regarding surgery and IPC were also collected. According to the surgical records, those who did not receive cytoreductive surgery were classified as the non-surgery group. Patients who had undergone complete resection of primary gastric lesions and cytoreductive surgery of PC were classified as the surgery group. Within the surgery group, complete resection surgery (CCR-0) indicated no remains of macroscopic residual cancer, whilst incomplete resection surgery (CCR-1) indicated residual nodules of PC. OS was calculated from the initial date of surgery, or first IPC, to mortality or the last recorded date of follow-up.

Statistics analysis. Kaplan-Meier methods were used to estimate the survival distributions stratified by pertinent clinical and histopathological variables. The log-rank test was used to perform univariate analysis of prognostic factors. Cox's proportional hazards regression model was used to perform multivariate analysis of prognostic predictors. $\mathrm{P}<0.05$ was considered to indicate a statistically significant difference. SPSS software, version 17 (SPSS Inc., Chicago, IL, USA) was used for all analyses.

\section{Results}

Patients and clinical data. A total of 57 patients presenting with advanced gastric cancer achieved the inclusion criteria, with a median age of 51 years. The median follow-up time was 12.4 months (range, 1-56 months). A total of 3 patients received IPC treatment alone, whilst 54 patients received IPC treatment alongside systemic chemotherapy. A total of 32 patients underwent cytoreductive surgery, with 11 achieving a CCR-0 resection and 21 achieving a CCR-1 resection. The other 25 non-surgery patients received either IPC alone or combined with systemic chemotherapy. Poorly-differentiated tumors were demonstrated to be common $(\sim 60 \%)$, whilst SRC adenocarcinoma accounted for $70.2 \%$ of all patients (40 patients). The median number of cycles of IPC administered was 3 (range, 1-6), whilst the median number of cycles of systemic chemotherapy administered was 5 (range, 1-27). A total of 20 patients (35.1\%) presented with symptomatic ascites at initial diagnosis (Table I).

OS. The median survival time for all 57 patients was calculated as 10.1 months [95\% confidence interval (CI), 6.8-13.5; Fig. 1]. The OS rates at 1,2 and 3 years were 46,19 and $12 \%$, respectively. In total, 4 of the 57 patients are currently alive without recurrence and had a median follow-up time of 33 months when the study finished. A total of 7 patients were lost to follow-up, whilst 5 patients survived for more than 3 years; all of these patients presented with no symptomatic ascites and received on average 12.6 cycles of systemic chemotherapy. Furthermore, 4 of these patients underwent a CCR-0 resection and only 1 demonstrated SRC adenocarcinoma.

Factors correlating with $O S$ in univariate analysis. A log-rank test was conducted to investigate the possible prognostic factors of OS. A total of 16 factors were analyzed, consisting of gender, primary disease site, ECOG PS score, symptomatic ascites, elevated tumor markers (CEA, CA19-9 and CA72-4), status of resection, histological subtype of adenocarcinoma, cycles of systemic chemotherapy, systemic chemotherapy containing oxaliplatin, fluorouracil or paclitaxel, cycles of IPC and IPC procedure containing cisplatin or 5-fluorouracil. Factors that favored an increased rate of survival (Table II) included an ECOG PS score of 0-1 [P=0.02; hazard ratio (HR), 1.91; 95\% CI, 1.09-3.35], no symptomatic ascites at primary diagnosis $(\mathrm{P}=0.005$; HR, 2.86; 95\% CI, 1.60-5.10), complete resection of all gross disease ( $\mathrm{P}=0.001$; HR, 2.74; 95\% CI, 1.79-4.22), non-schistosomal rectal cancer (nSRC) adenocarcinoma $(\mathrm{P}=0.037$; HR, 1.96; 95\% CI, 1.03-3.72), $\geq 4$ cycles of systemic chemotherapy $(\mathrm{P}=0.001 ; \mathrm{HR}, 1.67 ; 95 \% \mathrm{CI}, 0.15-0.48)$ and systemic chemotherapy containing oxaliplatin $(\mathrm{P}=0.01$; HR, 2.70; 95\% CI, 1.43-5.09) and fluorouracil ( $\mathrm{P}=0.006$; HR, 2.24; 95\% CI, 1.24-4.06). Systemic chemotherapy containing paclitaxel $(\mathrm{P}=0.07$; HR, 1.70; 95\% CI, 0.96-3.00) was demonstrated to be numerically, but not statistically different. Gender, primary disease site, elevated tumor markers (CEA, CA19-9 and CA72-4), cycles of IPC and IPC procedure containing cisplatin or 5-fluorouracil were not significantly associated with OS. 
Table I. Patient characteristics.

\begin{tabular}{|c|c|}
\hline Characteristics & Value \\
\hline Total patients, n (\%) & $57(100.0)$ \\
\hline Gender ratio (male:female), $\mathrm{n}$ & $29: 28$ \\
\hline Median age (range), years & $52.1(21-80)$ \\
\hline \multicolumn{2}{|l|}{ Tumor differentiation, n (\%) } \\
\hline High & $0(0.0)$ \\
\hline Moderate & $6(10.5)$ \\
\hline Poor & $34(59.7)$ \\
\hline Not reported & $17(29.8)$ \\
\hline \multicolumn{2}{|l|}{ Type of adenocarcinoma, n (\%) } \\
\hline $\mathrm{nSRC}$ & $17(29.8)$ \\
\hline $\mathrm{SRC}$ & $40(70.2)$ \\
\hline \multicolumn{2}{|l|}{ Symptomatic ascites, n (\%) } \\
\hline Yes & $20(35.1)$ \\
\hline No & $37(64.9)$ \\
\hline \multicolumn{2}{|c|}{ Performance status (ECOG), n (\%) } \\
\hline 0 & $2(3.5)$ \\
\hline 1 & $31(54.4)$ \\
\hline 2 & $24(42.1)$ \\
\hline \multicolumn{2}{|c|}{ Location of primary tumor, $\mathrm{n}(\%)$} \\
\hline Corpus & $26(45.6)$ \\
\hline Atrium & $19(33.3)$ \\
\hline Fundus & $10(17.6)$ \\
\hline Unknown & $2(3.5)$ \\
\hline \multicolumn{2}{|l|}{ Status of surgery, n (\%) } \\
\hline Resected (surgery) & $32(56.1)$ \\
\hline CCR-0 & $11(19.3)$ \\
\hline CCR-1 & $21(36.8)$ \\
\hline Undissected (non-surgery) & $25(43.9)$ \\
\hline \multicolumn{2}{|l|}{ Systemic chemotherapy, n (\%) } \\
\hline Paclitaxel-containing & $31(54.4)$ \\
\hline Oxaliplatin-containing & $42(73.7)$ \\
\hline Fluorouracil-containing & 37 (64.9) \\
\hline \multicolumn{2}{|l|}{ IPC agents, n (\%) } \\
\hline Cisplatin-containing & $43(75.4)$ \\
\hline 5-Fluorouracil-containing & $29(50.9)$ \\
\hline
\end{tabular}

Systemic chemotherapy and IPC agents percentages do not equal $100 \%$ as certain patients received more than one treatment. SRC, signet ring cell; nSRC, non-SRC; ECOG, Eastern Cooperative Oncology Group IPC; intraperitoneal chemotherapy.

Factors correlating with OS on multivariate analysis. A multivariate Cox regression was conducted to determine independent predictors of OS. Only four factors were demonstrated to be statistically different. Patients with no symptomatic ascites at surgery or primary diagnosis experienced a median OS time of 14.9 \pm 1.9 months compared with $4.2 \pm 2.4$ months for patients who did present with symptomatic ascites $(\mathrm{P}=0.003$; HR, 3.89; $95 \% \mathrm{CI}, 1.58-9.64)$. The median OS time was $14.9 \pm 1.3$ months for patients who underwent surgery, and $6.3 \pm 2.3$ months

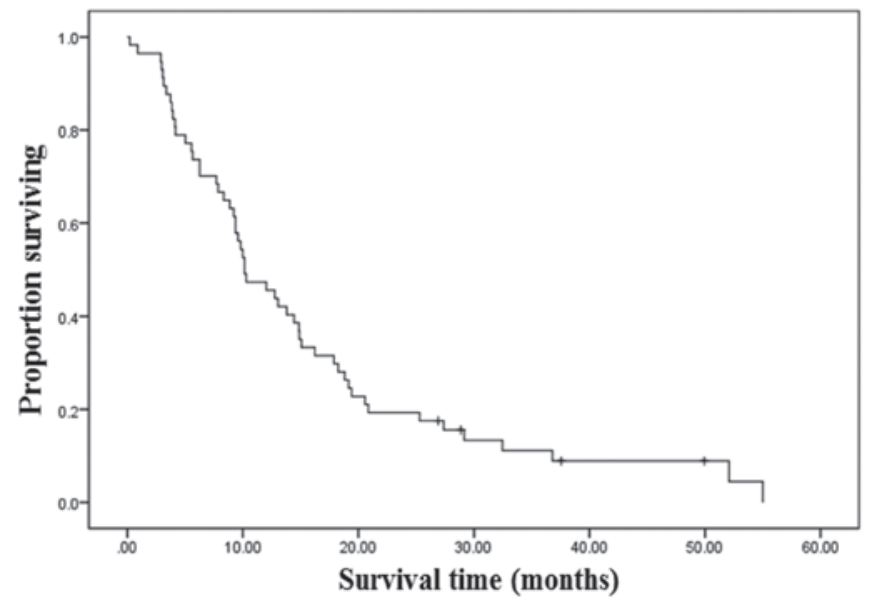

Figure 1. OS of all 57 patients, with a median OS time of 10.1 months. The OS rate at 1,2 and 3 years was 46, 19 and $12 \%$, respectively. OS, overall survival.

for those who did not undergo surgery $(\mathrm{P}=0.010$; HR, 3.49; 95\% CI, 1.34-9.04). For patients with a CCR-0 resection, the median survival time was 32.5 months in comparison to $13.8 \pm 1.3$ months for those with a CCR-1 resection. Patients with $\mathrm{nSRC}$ adenocarcinoma experienced a median OS time of 17.9 \pm 5.6 months, which was longer than the $9.8 \pm 0.6$ months observed in patients with $\mathrm{SRC}$ adenocarcinoma $(\mathrm{P}=0.024$; HR, 2.94, 95\% CI, 1.15-7.47). Those individuals who received $\geq 4$ cycles of systemic chemotherapy experienced a median OS time of $17.9 \pm 3.6$ months, while patients who had received $<4$ cycles of systemic chemotherapy only experienced a median OS time of 5.6 \pm 1.4 months $(\mathrm{P}=0.001$; HR, 0.13; 95\% CI, 0.06-0.32) (Table III and Fig. 2).

Adverse effects. The grade 3 to 4 treatment-related adverse effects morbidity rate was $22.8 \%$ (13 patients). Severe bone marrow suppression was noted in 8 patients (14.3\%), severe nausea and vomiting in 3 patients (5.3\%), and severe diarrhea in 2 patients $(3.5 \%)$. Obstruction of the digestive tract was observed in 5 patients $(8.8 \%)$ and all instances were considered as progression of the disease rather than as the adverse effects of IPC. Puncture-associated complaints were primarily mild distention and abdominal pain. Peri-operative mortality (mortality during the 3 months after surgery) in the patients who underwent surgery was $3.1 \%$ (1 patient), with this patient succumbing 87 days after surgery.

\section{Discussion}

PC originating from gastric cancer is a prevalent disease, which occurs in 5-20\% of patients being assessed for potentially curative resection and accounts for more than half of the mortalities in those patients $(11,16)$. Patients presenting with $\mathrm{PC}$ have a relatively short survival time, yet there is not a standard treatment for the disease $(12,17)$. Currently, PC that originates from gastric cancer is typically treated with the use of palliative systemic chemotherapy only, however, the literature regarding the efficacy of this treatment when used alone is limited, with a recorded median OS time of only 6 months (5). In the present study, patients undergoing systematic chemotherapy, including 
Table II. Survival by univariate analysis.

\begin{tabular}{|c|c|c|c|c|c|c|c|c|c|}
\hline \multirow[b]{2}{*}{ Characteristics } & \multirow[b]{2}{*}{$\mathrm{n}$} & \multirow{2}{*}{$\begin{array}{l}\text { Median survival } \\
\text { time, months }\end{array}$} & \multicolumn{4}{|c|}{ Years, n (\%) } & \multirow{2}{*}{$\begin{array}{l}\text { Hazard } \\
\text { ratio }\end{array}$} & \multirow[b]{2}{*}{$95 \% \mathrm{CI}$} & \multirow[b]{2}{*}{$\mathrm{P}$-value } \\
\hline & & & 0.5 & 1 & 2 & 3 & & & \\
\hline PS score & & & & & & & 1.91 & $1.09-3.35$ & 0.02 \\
\hline $0-1$ & 33 & $12 \pm 2.3$ & $28(84.8)$ & $16(48.5)$ & $10(30.3)$ & $4(12.1)$ & & & \\
\hline 2 & 24 & $9.2 \pm 2.8$ & $14(58.3)$ & $10(41.7)$ & $1(4.2)$ & $1(4.2)$ & & & \\
\hline Resection status & & & & & & & 2.74 & $1.79-4.22$ & 0.001 \\
\hline Surgery & 32 & $14.9 \pm 1.3$ & $29(90.6)$ & $22(68.8)$ & $11(34.3)$ & $5(15.6)$ & & & \\
\hline CCR-0 & 11 & $32.4 \pm 10.9$ & $11(100.0)$ & $9(81.8)$ & $6(54.5)$ & $4(36.4)$ & & & \\
\hline CCR-1 & 21 & $13.8 \pm 1.3$ & $18(85.7)$ & $13(61.9)$ & $5(23.8)$ & $1(4.76)$ & & & \\
\hline Non-surgery & 25 & $6.3 \pm 2.3$ & $13(52.0)$ & $4(16.0)$ & $0(0.0)$ & $0(0.0)$ & & & \\
\hline Symptomatic ascites & & & & & & & 2.86 & $1.60-5.10$ & 0.005 \\
\hline Yes & 20 & $4.2 \pm 2.4$ & $10(50.0)$ & $3(15.0)$ & $1(5.0)$ & $0(0.0)$ & & & \\
\hline No & 37 & $14.9 \pm 1.9$ & $32(86.5)$ & $23(62.2)$ & $10(27.0)$ & $5(13.5)$ & & & \\
\hline Type of adenocarcinoma & & & & & & & 1.96 & $1.03-3.72$ & 0.037 \\
\hline SRC & 40 & $9.8 \pm 0.6$ & $29(72.5)$ & $17(42.5)$ & $5(12.5)$ & $1(2.5)$ & & & \\
\hline $\mathrm{nSRC}$ & 17 & $17.9 \pm 5.6$ & $13(76.5)$ & $9(52.9)$ & $6(35.3)$ & $4(23.5)$ & & & \\
\hline \multicolumn{10}{|l|}{ Systemic chemotherapy } \\
\hline Oxaliplatin-containing & & & & & & & 2.70 & $1.43-5.09$ & 0.01 \\
\hline Yes & 42 & $12.8 \pm 1.8$ & $35(83.3)$ & $23(54.8)$ & $11(26.2)$ & $5(11.9)$ & & & \\
\hline No & 15 & $5.6 \pm 1.5$ & $7(46.7)$ & $4(26.7)$ & $0(0.0)$ & $0(0.0)$ & & & \\
\hline Fluorouracil-containing & & & & & & & 2.24 & $1.24-4.06$ & 0.006 \\
\hline Yes & 37 & $12.8 \pm 2.2$ & $28(75.7)$ & $19(51.4)$ & $10(27.0)$ & $5(13.5)$ & & & \\
\hline No & 20 & $8.3 \pm 3.9$ & $11(55.0)$ & $7(35.0)$ & $1(5.0)$ & $0(0.0)$ & & & \\
\hline $\begin{array}{l}\text { Cycles of systemic } \\
\text { chemotherapy }\end{array}$ & & & & & & & 1.67 & $0.15-0.48$ & 0.001 \\
\hline$\geq 4$ & 29 & $17.9 \pm 3.6$ & $29(100.0)$ & $20(69.0)$ & $10(34.5)$ & $4(13.8)$ & & & \\
\hline$<4$ & 28 & $5.6 \pm 1.4$ & $13(46.4)$ & $6(21.4)$ & $1(3.6)$ & $1(3.6)$ & & & \\
\hline
\end{tabular}

Survival data categorized by clinicopathological factors identified as statistically significant in a log-rank test. P-values are based on the data from the log-rank test. CI, confidence interval; SRC, signet ring cell; nSRC, non-SRC.

Table III. Independent predictive clinicopathologic factors in Cox's regression.

\begin{tabular}{lccr}
\hline & & \multicolumn{2}{c}{$95 \%$ CI } \\
\cline { 2 - 4 } Parameters & Hazard ratio & Lower & Upper \\
\hline Symptomatic ascites (yes vs. no) & 3.89 & 1.58 & 9.64 \\
Status of resection (CCR-1 vs. CCR-0) & 3.49 & 1.34 & 9.04 \\
Type of adenocarcinoma (SRC vs. nSRC) & 2.94 & 1.15 & 7.47 \\
Cycles of systemic chemotherapy ( $\geq 4$ vs. $<4)$ & 0.13 & 0.06 & 0.010 \\
\hline
\end{tabular}

CI, confidence interval; CCR-0, complete resection surgery; CCR-1, incomplete resection surgery; SRC, signet ring cell. nSRC, non-SRC.

a proportion of patients who did not receive surgery (43.9\%), demonstrated a median OS time of 10.1 months. Although certain studies have reported longer survival times, metachronism PC was also included in those studies, possibly skewing the results $(18,19)$; however, in the current study, all patients presented with PC at primary diagnosis or during surgery.
Considering this, the median OS time of 10.1 months established in the present study was a relatively long survival time when compared with published data from other studies investigating patients with PC originating from gastric cancer.

In the present study, SRC adenocarcinoma was confirmed as an independent factor associated with a reduced OS time in 

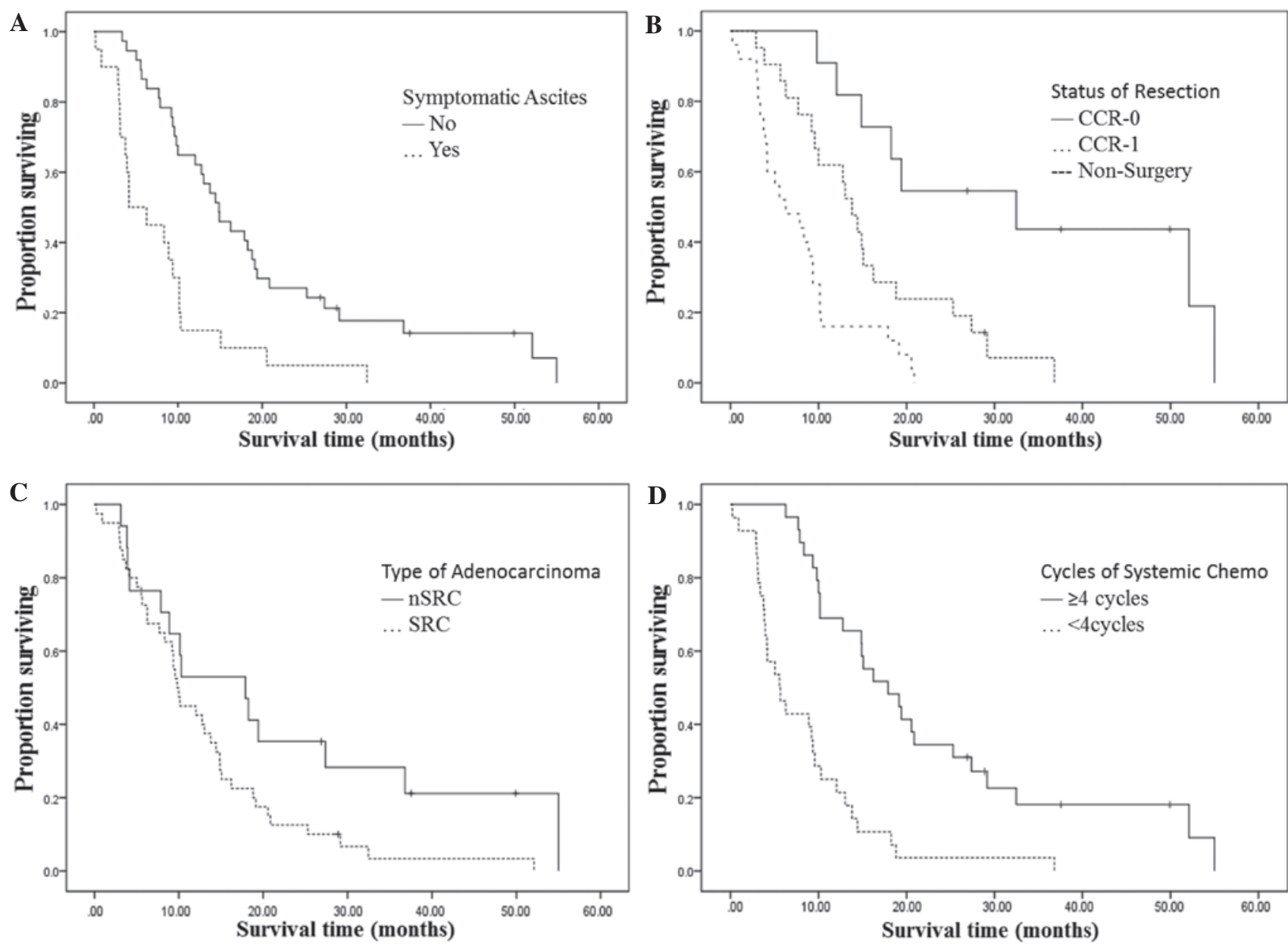

Figure 2. Survival curves are illustrated for patients with different clinicopathological factors. (A) Survival of patients who presented with symptomatic ascites (volume $\geq 500 \mathrm{ml}$ ) or without symptomatic ascites at surgery or primary diagnosis. (B) Survival of patients who underwent CCR-0 and CCR-1, or those without surgery (non-surgery). (C) Survival of patients with nSRC or SRC. (D) Survival of patients who received $\geq 4$ or $<4$ cycles of systemic chemotherapy. CCR-0, complete resection surgery; CCR-1, incomplete resection surgery; SRC, signet ring cell adenocarcinoma; nSRC, non-SRC.

PC patients who had been treated with IPC. Similar results have been demonstrated in tumors with gynecological and colorectal origins $(20,21)$. In the present study, the median OS time was 18.3 months for patients with nSRC adenocarcinoma compared with 9.8 months for patients with SRC. SRC adenocarcinoma was present in $70.2 \%$ of all patients in the current study, whilst only accounting for 10.2-15.6\% of all gastric cancer cases globally $(22,23)$. This could be explained by the idea that SRC adenocarcinoma metastasizes earlier during development, therefore causing PC to arise more frequently than nSRC adenocarcinoma. SRC adenocarcinoma of the stomach is associated with an improved prognosis when compared with nSRC adenocarcinoma in early gastric cancer, however, it is associated with a poor prognosis in comparison to nSRC adenocarcinoma at an advanced disease stage $(22,24)$. A further reason for the poor prognosis associated with SRC may be that SRC adenocarcinoma appears to be more resistant to currently used cytotoxic or biological agents, with more frequent drug resistance-associated gene mutations observed in SRC adenocarcinoma $(25,26)$.

In the present study, resection status was one of the most significant prognostic factors for survival $(\mathrm{P}<0.001)$ as determined by multivariate analysis. The median OS time was observed as $14.9 \pm 1.3$ months for patients who underwent surgery, and $6.3 \pm 2.3$ months for those who did not undergo surgery
$(\mathrm{P}=0.010)$. Similar results were reported by Scaringi et al (3), with a median survival time of 6.6 months in patients with PC originating from gastric cancer who had been treated by cytoreductive surgery and hyperthermia IPC. Glehen et al (11) also reported a median survival time of 9.2 months in 159 patients who had been treated by cytoreductive surgery combined with perioperative IPC. The effect of IPC treatment on survival when used alone in patients with PC originating from gastric cancer has not yet been reported. In the present study, the median OS time was only $6.3 \pm 2.3$ months for those with undissected primary tumors, similar to that observed in patients with PC who received only systemic chemotherapy (median OS time, 6 months) (5). The synergistic effects of cytoreductive surgery to remove the macroscopic tumor and of IPC to eradicate microscopic residual diseases are major advantages of this combined approach. In the surgery group from the current study, the median OS time for CCR-0 patients $(32.4 \pm 10.9$ months) was considerably higher than that demonstrated for the CCR-1 patients (13.8 \pm 1.3 months). Similar results were reported by Yonemura et al in a study of 107 patients; the median survival time after complete cytoreduction was 15.5 months, and the median survival time following incomplete cytoreduction was 7.9 months (12). Glehen et al conducted an analysis of 159 patients from 15 institutions. For patients treated by 
CCR-0 surgery, the median survival time was 15 months, compared with 6 or 4 months for CCR-1 or CCR-2 patients, respectively (11). Even in patients where a CCR-0 cannot be undertaken, a nearly complete resection or CCR-1 can still achieve a longer survival time compared with that of patients with large residual tumor nodules left (10). This implies that a more radical surgery strategy may be taken into consideration. However, a thorough and precise assessment prior to surgery should be addressed (27).

Symptomatic ascites is an independent factor associated with poor survival. In the present study, patients with ascites at primary diagnosis or surgery experienced a median survival time of 4.2 months, compared with 14.9 months for patients without the presence of symptomatic ascites. Numerous studies have reported a median survival time of 4-33 weeks for patients presenting with symptomatic ascites (28). This therefore indicates that ascites should be included in the regular evaluation prior to cytoreductive surgery in order to make a fully informed judgment. Symptomatic ascites often complicates PC and may severely impair quality of life (29). However, despite the improvement of survival in those patients being limited, IPC has demonstrated a high effectiveness in symptom control, with it being reported that $>80 \%$ of ascites was controlled following manipulation $(30,31)$. This was observed typically following the use of conventional treatments, including repeated paracenteses, diuretics and systemic chemotherapy. However, although they may initially be successful, these therapeutics strategies lose their efficacy over time, so cannot be considered as a long-term fix (29).

Systemic chemotherapy has been regarded as an effective treatment strategy for patients with gastric cancer undergoing multidisciplinary treatment. Partial and complete responses following systemic chemotherapy for PC of gastric origin have been reported $(32,33)$. In the present study, 54 patients (94.7\%) received systemic chemotherapy. Those who had the chance to receive $\geq 4$ cycles of systemic chemotherapy experienced a median OS time of $17.9 \pm 3.6$ months. This was a significant improvement in comparison to patients who received $<4$ cycles $(5.6 \pm 1.4$ months) $(\mathrm{P}=0.001)$. As previously mentioned, systemic chemotherapy alone did not appear to produce a significant improvement in the OS of gastric cancer patients with PC (5). Regarding the results of the current study, it is believed that on the basis of local control of IPC, systemic chemotherapy may be an important modality for survival benefits. The results of the current study also indicate that the use of systemic chemotherapy should not be ceased too early into treatment without the observation of disease progression or evidence of resistance. However, regarding the evidence that increased cycles of systemic chemotherapy may be the consequence of a better response for chemotherapy, further investigation is required to confirm its effect.

It should be noted that the present study was conducted with a small sample size, and the differing regimens of chemotherapy and IPC agents have made the investigation difficult. Despite this, to the best of our knowledge, the current study is the first analysis that evaluates the effect of IPC on survival in patients with PC originating from gastric cancer, as well as evaluating the correlation of patient characteristics with OS. We believe that the results from the present study may provide important information that may aid in clinical practice for patients with PC.

In conclusion, IPC is one of the most important treatments administered to patients presenting with the peritoneal spread of gastric cancer. A complete resection of all gross disease, receiving an increased number of cycles of systemic chemotherapy ( $\geq 4$ cycles) and certain clinical pathological factors, including nSRC adenocarcinoma and no symptomatic ascites, are all advantageous factors for an extended OS time in PC patients.

\section{References}

1. Yan TD, Black D, Savady R and Sugarbaker PH: Systematic review on the efficacy of cytoreductive surgery combined with perioperative intraperitoneal chemotherapy for peritoneal carcinomatosis from colorectal carcinoma. J Clin Oncol 24: 4011-4019, 2006.

2. Gill RS, Al-Adra DP, Nagendran J, Campbell S, Shi X, Haase E and Schiller D: Treatment of gastric cancer with peritoneal carcinomatosis by cytoreductive surgery and HIPEC: A systematic review of survival, mortality, and morbidity. J Surg Oncol 104: 692-698, 2011.

3. Scaringi S, Kianmanesh R, Sabate JM, Sabate JM, Facchiano E, Jouet P, Coffin B, Parmentier G, Hay JM, Flamant Y and Msika S: Advanced gastric cancer with or without peritoneal carcinomatosis treated with hyperthermic intraperitoneal chemotherapy: A single western center experience. Eur J Surg Oncol 34: 1246-1252, 2008.

4. Chu DZ, Lang NP, Thompson C, Osteen PK and Westbrook KC: Peritoneal carcinomatosis in nongynecologic malignancy. A prospective study of prognostic factors. Cancer 63: 364-367, 1989.

5. Wagner AD, Grothe W, Haerting J, Kleber G, Grothey A and Fleig WE: Chemotherapy in advanced gastric cancer: A systematic review and meta-analysis based on aggregate data. J Clin Oncol 24: 2903-2909, 2006.

6. Cotte E, Colomban O, Guitton J, Tranchand B, Bakrin N, Gilly FN, Glehen O and Tod M: Population pharmacokinetics and pharmacodynamics of cisplatinum during hyperthermic intraperitoneal chemotherapy using a closed abdominal procedure. J Clin Pharmacol 51: 9-18, 2011.

7. Rossi CR, Mocellin S, Pilati P, Foletto M, Quintieri L, Palatini P and Lise M: Pharmacokinetics of intraperitoneal cisplatin and doxorubicin. Surg Oncol Clin N Am 12: 781-794, 2003.

8. Chua TC, Robertson G, Liauw W, Farrell R, Yan TD and Morris DL: Intraoperative hyperthermic intraperitoneal chemotherapy after cytoreductive surgery in ovarian cancer peritoneal carcinomatosis: Systematic review of current results. J Cancer Res Clin Oncol 135: 1637-1645, 2009.

9. Esquivel J, Elias D, Baratti D, Kusamura S and Deraco M: Consensus statement on the loco regional treatment of colorectal cancer with peritoneal dissemination. J Surg Oncol 98: 263-267, 2008.

10. Yang XJ, Li Y and Yonemura Y: Cytoreductive surgery plus hyperthermic intraperitoneal chemotherapy to treat gastric cancer with ascites and/or peritoneal carcinomatosis: Results from a Chinese center. J Surg Oncol 101: 457-464, 2010.

11. Glehen O, Gilly FN, Arvieux Cotte E, Boutitie F, Mansvelt B, Bereder JM, Lorimier G, Quenet F and Elias D; Association Française de Chirurgie: Peritoneal carcinomatosis from gastric cancer: A multi-institutional study of 159 patients treated by cytoreductive surgery combined with perioperative intraperitoneal chemotherapy. Ann Surg Oncol 17: 2370-2377, 2010.

12. Yonemura Y, Kawamura T, Bandou E, Takahashi S, Sawa T and Matsuki N: Treatment of peritoneal dissemination from gastric cancer by peritonectomy and chemohyperthermic peritoneal perfusion. Br J Surg 92: 370-375, 2005.

13. Glehen O, Gilly FN, Boutitie F, Bereder JM, Quenet F, Sideris L, Mansvelt B, Lorimier G, Msika S and Elias D; French Surgical Association: Toward curative treatment of peritoneal carcinomatosis from nonovarian origin by cytoreductive surgery combined with perioperative intraperitoneal chemotherapy: A multi-institutional study of 1,290 patients. Cancer 116: 5608-5618, 2010. 
14. Shen P, Hawksworth J, Lovato J, Loggie BW, Geisinger KR, Fleming RA and Levine EA: Cytoreductive surgery and intraperitoneal hyperthermic chemotherapy with mitomycin $\mathrm{C}$ for peritoneal carcinomatosis from nonappendiceal colorectal carcinoma. Ann Surg Oncol 11: 178-186, 2004.

15. Oken MM, Creech RH, Tormey DC, Horton J, Davis TE, McFadden ET and Carbone PP: Toxicity and response criteria of the Eastern Cooperative Oncology Group. Am J Clin Oncol 5: 649-655, 1982.

16. Jemal A, Bray F, Center MM, Ferlay J, Ward E and Forman D: Global cancer statistics. CA Cancer J Clin 61: 69-90, 2011.

17. Yoo CH, Noh SH, Shin DW, Choi SH and Min JS: Recurrence following curative resection for gastric carcinoma. Br J Surg 87: 236-242, 2000.

18. Cashin PH, Graf W, Nygren P and Mahteme H: Intraoperative hyperthermic versus postoperative normothermic intraperitoneal chemotherapy for colonic peritoneal carcinomatosis: A case-control study. Ann Oncol 23: 647-652, 2012.

19. Cavaliere F, De Simone M, Virzi S, Deraco M, Rossi CR, Garofalo A, Di Filippo F, Giannarelli D, Vaira M, et al: Prognostic factors and oncologic outcome in 146 patients with colorectal peritoneal carcinomatosis treated with cytoreductive surgery combined with hyperthermic intraperitoneal chemotherapy: Italian multicenter study S.I.T.I.L.O. Eur J Surg Oncol 37: 148-154, 2011.

20. Shen P, Levine EA, Hall J, Case D, Russell G, Fleming R, McQuellon R, Geisinger KR and Loggie BW: Factors predicting survival after intraperitoneal hyperthermic chemotherapy with mitomycin $\mathrm{C}$ after cytoreductive surgery for patients with peritoneal carcinomatosis. Arch Surg 138: 26-33, 2003.

21. Cao C, Yan TD, Black D and Morris DL: A systematic review and meta-analysis of cytoreductive surgery with perioperative intraperitoneal chemotherapy for peritoneal carcinomatosis of colorectal origin. Ann Surg Oncol 16: 2152-2165, 2009.

22. Kunisaki C, Shimada H, Nomura M, Matsuda G, Otsuka Y and Akiyama $\mathrm{H}$ : Therapeutic strategy for signet ring cell carcinoma of the stomach. Br J Surg 91: 1319-1324, 2004.

23. Otsuji E, Yamaguchi T, Sawai K and Takahashi T: Characterization of signet ring cell carcinoma of the stomach. J Surg Oncol 67: 216-220, 1998.

24. Kim DY, Park YK, Joo JK, Ryu SY, Kim YJ, Kim SK and Lee JH: Clinicopathological characteristics of signet ring cell carcinoma of the stomach. ANZ J Surg 74: 1060-1064, 2004.
25. Chu PG and Weiss LM: Immunohistochemical characterization of signet-ring cell carcinomas of the stomach, breast, and colon. Am J Clin Pathol 121: 884-892, 2004.

26. Nakatsuru S, Yanagisawa A, Ichii S, Tahara E, Kato Y, Nakamura Y and Horii A: Somatic mutation of the APC gene in gastric cancer: Frequent mutations in very well differentiated adenocarcinoma and signet-ring cell carcinoma. Hum Mol Genet 1: 559-563, 1992.

27. Hall JJ, Loggie BW, Shen P, Beamer S, Douglas Case L, McQuellon R, Geisinger KR and Levine EA: Cytoreductive surgery with intraperitoneal hyperthermic chemotherapy for advanced gastric cancer. J Gastrointest Surg 8: 454-463, 2004.

28. Loggie BW, Fleming RA, McQuellon RP, Russell GB and Geisinger KR: Cytoreductive surgery with intraperitoneal hyperthermic chemotherapy for disseminated peritoneal cancer of gastrointestinal origin. Am Surg 66: 561-568, 2000.

29. Facchiano E, Scaringi S, Kianmanesh R, Sabate JM, Castel B, Flamant Y, Coffin B and Msika S: Laparoscopic hyperthermic intraperitoneal chemotherapy (HIPEC) for the treatment of malignant ascites secondary to unresectable peritoneal carcinomatosis from advanced gastric cancer. Eur J Surg Oncol 34: $154-158,2008$

30. Sayag AC, Gilly FN, Carry PY, Perdrix JP, Panteix G, Brachet A, Banssillon V and Braillon G: Intraoperative chemohyperthermia in the management of digestive cancers. A general review of literature. Oncology 50: 333-337, 1993.

31. Gilly F, Carry P, Bracket A, Sayag AC, Panteix G, Salle B, Bienvenu J, Banssillon V, Burgard G, Manchon M, et al: Treatment of malignant peritoneal effusion in digestive and ovarian cancer. Med Oncol Tumor Pharmacother 9: 177-181, 1992.

32. Inokuchi M, Yamashita T, Yamada H, Kojima K, Ichikawa W, Nihei Z, Kawano T and Sugihara K: Phase I/II study of S-1 combined with irinotecan for metastatic advanced gastric cancer. Br J Cancer 94: 1130-1135, 2006.

33. Yano M, Shiozaki H, Inoue M, Tamura S, Doki Y, Yasuda T, Fujiwara Y, Tsujinaka T and Monden M: Neoadjuvant chemotherapy followed by salvage surgery: Effect on survival of patients with primary noncurative gastric cancer. World J Surg 26: 1155-1159, 2002. 